\title{
Inter-Collateral Diabetic Retinopathy Extraction and Evaluation Using Trained Datasets of Multiple Feature Set
}

\author{
K.T.Ilayarajaa1, E.Logashanmugam ${ }^{2}$ \\ ${ }^{1}$ Research Scholar, Faculty of Electronics Engineering, Sathyabama Institute of Science and \\ Technology, Chennai, India. \\ ${ }^{2}$ Professor, Department of Electronics and Communication Engineering, Sathyabama Institute \\ of Science and Technology, Chennai, India. \\ ilayarajaa.tsi@gmail.com ${ }^{1}, \operatorname{logu} 999 @$ gmail.com ${ }^{2}$
}

Article History: Received: 10 November 2020; Revised 12 January 2021 Accepted: 27 January 2021; Published online: 5 April 2021

\begin{abstract}
Diabetic Retinopathy (DR) is caused due to un-mounted diabetic comorbidities. The patients suffer complete vision blindness if untreated or diagnosed on later stage. In this article, we propose a novel approach for early detection and prediction using trained datasets of multiple features. The process expansion is resultant of multiple stage attribute extraction via a series of inter-collateral parameters of diabetics. Typically, the proposed technique is designed and developed on a multi-value and multi-dimension datasets such as comorbidities history of patient encountered during diabetics. The proposed technique uses collateral attributes in evaluating retinopathy status and thereby validates the extracted DR under threshold value comparisons. The results are computed using HADOOP framework for recursive pattern and feature evaluation. The trial is processed on UCL digital library datasets with estimated performance of $98.7 \%$ with extraction and $92.34 \%$ with value True-Positive (TP) prediction.
\end{abstract}

Keywords - Diabetic Retinopathy, Feature extraction, multi-dimensional dataset evaluation, intercollateral attribute extractions.

\section{INTRODUCTION}

The diabetic retinopathy (DR) is a regular formation of extra-perianal growth in the inner region of human lens causing a permanent or complete blindness if untreated in early stages. The main reason for its occurrence is due to diabetics mellitus. According to a study [1] 72.96 million cases is reported in India as per the WHO survey. This causes a major concern of research and development to address the growing demand of treatment and drug design for developing countries population. The current scenario of diabetic retinopathy is relatively pushed on back-foot due to the break of Global pandemic SAR CoVID-19 in late December of 2019 reported from Wuhan province of China. The concern of diabetic's retinopathy and related illness caused due to diabetics is rising on an alarming rate. In this article, a study is focused on the design and development of a technique dedicated for the 
feature extraction and evaluation of DR based on a predictive model. The process of decision making is relatively incubated with an ideal condition of attribute interdependency extraction.

The research focus is on the collateral attributes extractions. These attributes are functional parameters of diabetic retinopathy based datasets. The technique highlights attribute interdependency such as age of patients, sample size of DR, medical samples and patient's history in cause of DR based on occurrence and inheritance. The research in this article is presented with the following orientation. The survey and current existing terminologies are discussed in session 2 i.e. literature survey, followed by the methodology and block diagram of proposed system. The mathematical model of proposed technique is demonstrated in session 4 and results and discussions are further discussed in session 5 and 6 respectively.

\section{LITERATURE SURVEY}

The diagnosis and treatment via systematic screening of diabetic mellitus is processed and validated by various researchers and product design companies, whereas the challenge still remains in structural identification and process automation of the diabetic comorbidities ill'ment prediction and categorization. In [2] the authors have discussed various classification approaches of diabetic mellitus and particularly focused on the classification of diabetic retinopathy. The study has discussed the classification based on the ratio of 4:2:1 for diabetic retinopathy (DR) with reference to diabetic mellitus. In the early of 1989, a study [3] on retinopathy inclusion is proposed with various types of phonographic standards and its influence in classifying diabetic retinopathy. This study manually details with 2694 active diabetic patients and concluded the impact of DR classification.

Screening of diabetic retinopathy is a major challenge. The initial proposed approach is based on Computer Aided Images (CAI) [4]. These images were further used in classifying the approaches of retinopathy (DR) into the priority of treatment using trivial medical images and medical records such as X-Rays. With further development of technology, the modern approaches of classification and detection such as [5] is proposed. The [5] is based on microaneurysms (MA's) classification and segregation using SVM and GMM techniques. These techniques provided a new milestone in detection and decision making of biomedical samples until AI and ML was not introduced

The images of diabetic retinopathy samples were digitalized and hence, the remote transfer and layer compressing was at major challenge. The process scheme of image compression discussed in [6] is future extended and developed for improvisation under remote transmission as the proposed technique is based on collateral attributes extractions. The process of compression assures the images are secure while transmission with respect to the attributes and features associated with pixel density, ratio and space. The process of compression is supported by [7] based on Deep Learning (DL) approach. The study has provided supportive results in distancing various categories of retinopathy. The terminology of single medical dataset is not reduced to support multi-dimensional dataset processing. The [8] proposes a MooM datasets for processing the basic medical data types such as images, 
signals and records. In this proposed technique, the agenda of co-lateral attributes extraction is supported and developed on multi-dimensional datasets, collected from various resources and libraries.

\section{METHODOLOGY AND DESIGN}

The proposed system is designed with a focus to extract process and provide a decision support in treatment and detection of diabetic retinopathy (DR). The proposed technique is supported on multi-dimensional data processing scheme as shown in Fig. 1. The process is termed as an offline processing approach, with a defined raw datasets. These datasets are collected from various resources, independent of data-type, data-dimension and data-size. The originality of data is preserved and thus supported for collateral attribute extraction. The process uses a MooM [8] technique for standardization of raw datasets post-pre-processing. The standardization approach is to assure the data collected from various sources is aligned as per the processing and computation standards.

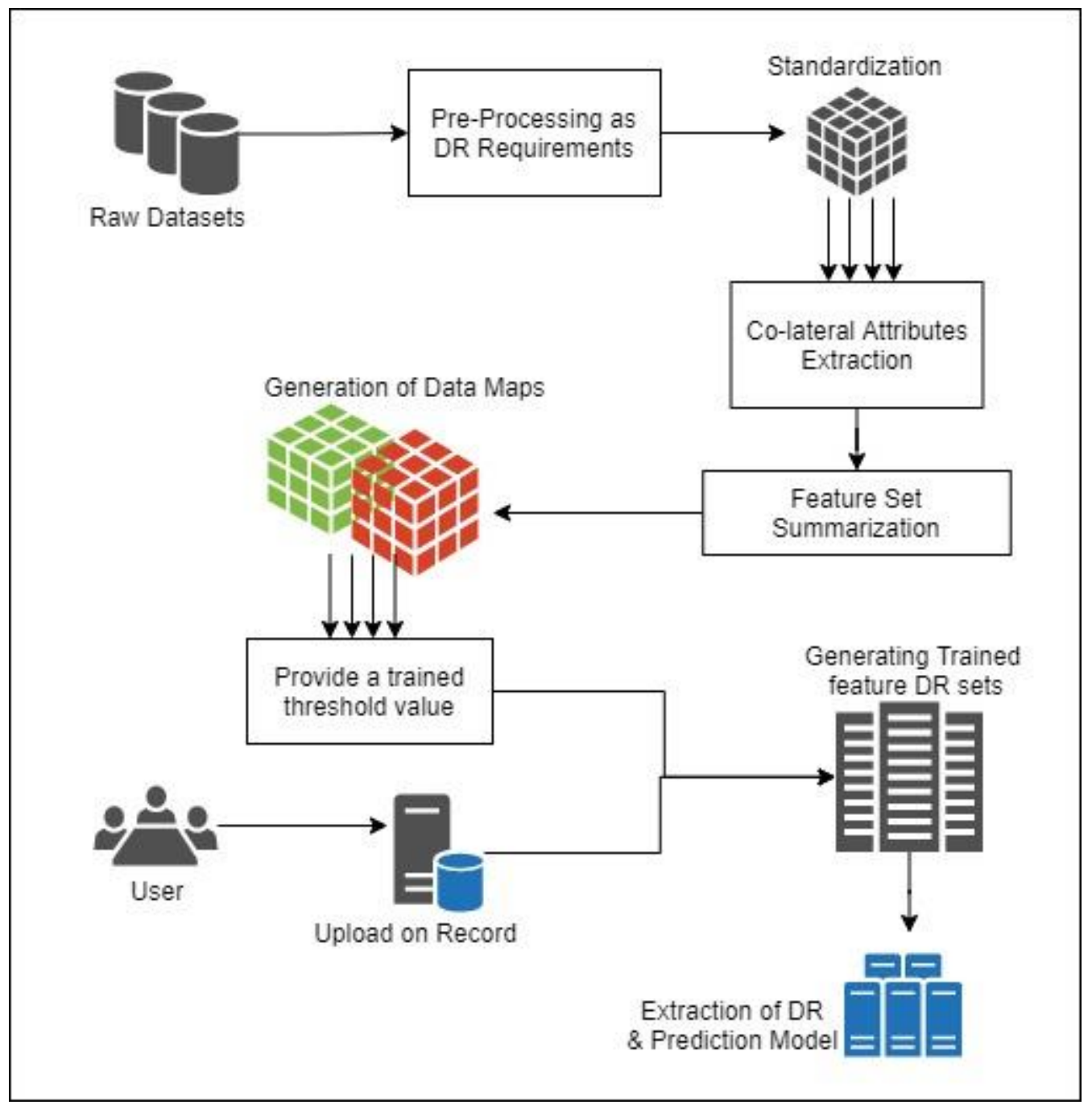

Fig. 1: Methodology block diagram of proposed co-lateral attribute extraction technique of DR decision support 
The data from standardization is processed with a Collateral Attribute Extraction Unit (CAE). This unit generates a systematic approach of defining attributes with respect to interdependency identification and mapping. The attribute dependencies play a vital role in concluding the co-relation of features set summarization. The Features set is typically, a onestop solution for processed datasets (DR) towards generation of data maps. These data-maps are functional units of proposed technique. It supports multi-dimensional data paring and attribute attainment. The process generates a threshold value of dataset array in-terms of collateral parameter.

The user information is collected from a dynamic front and processed under a single umbrella of trained feature datasets unit. This unit assures the parameters to interconnect with supporting SVM attributes via a user input and thus generates a decision support. The decision is a summarized evaluation of prediction model and extraction values.

\section{MATHEMATICAL REPRESENTATION OF CAE TECHNIQUE}

Diabetic retinopathy (DR) extraction and evaluation requires information processing with respect to data monitoring system. The proposed system of co-lateral attributes extraction based DR decision making results in a systematic mined tools of evaluation. The process is supported by a standardization mechanism of MooM datasets [7] as primary pre-processing techniques. The approach is supported under three steps, the primary is extraction of collateral attributes and feature standardization. The second is generation of data maps and system thersholding with reference to value charting. The third and final step of processing is extracting and evaluating the prediction model of occurrence of diabetic retinopathy with a comparative model of evaluation and training.

Consider the training set $(T)$ of diabetic retinopathy $(D R)$ from a computed module of dataattributes as $\left(A_{1}, A_{2}, A_{3} \ldots A_{n}\right)$ such that, each of attribute set is co-related and mapped to structured data-parameters of $\mathrm{T}$ as $T=\left[\left(P a_{1}, P a_{2}, P a_{3} \ldots P a_{n},\right) / \forall P a_{i} \in T \mathrm{U} T \subseteq A\right]$. These attributes are intended to reflect the variable mapping of each attributes inter-dependencies. The process of DR-requirement extraction is evaluated and reported in a standardization of datasets.

These datasets process inter-dependent attributes of each DR datasets such as the nonrepeated data parameters are reflected and optimized. The process of standardization is supported using multi-dimensional optimal medical datasets or MooM datasets. The each extracted attributes are reflected and marked with reference to value paradigms. The main goals of proposed systems is to extract the co-lateral attributes of DR as shown in Eq. 1

$T=\log _{2} \partial(x)_{i}\left[\sum_{j}^{n} \frac{\partial(P a)_{j}}{\partial t}\right]_{i=o}^{\infty}$ 
Where, the $\left(x_{i}\right)$ is represents information set of inter-dependent attributes with reference to parametric evaluation matrix $(\mathrm{Pa})$ under the condition set of $\left(T \subseteq P a / \forall P a_{i} \in A\right)$ where (A) represents attribute set of correlated parameters in diabetic retinopathy. The updating collateral of each $(T)$ is demonstrated in Eq. 2 and Eq. 3 respectively.

$T=\log _{2} \partial\left(x_{i}\right)\left[\lim _{n \rightarrow \infty}\left(\sum_{j}^{n} \frac{\partial(P a)_{j}}{\partial t}\right)\right]$

$T=\log _{2} \partial\left(x_{i}\right)\left[\lim _{n \rightarrow \infty}\left\{\sum_{j=0}^{n}\left(\sum_{k=j}^{n-1} \frac{\partial(P a)_{j} \cdot \partial(T)_{k}}{\partial t}\right) \partial x\right\} \Delta t\right]$

The attributes set of $(\mathrm{T})$ from Eq. 3 represents information extracted of inter-dependent attributes of DR such that, $\left(\forall\left[P a_{i}\right] \subset T\right)$ these attributes set of variables are further corelated and computed with a constant $\log _{2} \partial\left(x_{i}\right)$ variable as threshold variables. The computational matrix of trained sets $(T)$ is summarized via a recorded feature set $(f)$. this set is also relates the attribute of Eq. 3 to formulate the regional matrix values as shown in Eq. 4 and Eq. 5 respectively.

$$
f=\frac{\partial(T)}{\partial(P)} \forall\left(T_{i} \subseteq P \mathrm{U} P \in \lim _{n \rightarrow \infty}\left[\frac{\partial\left(x_{i}\right)_{n}}{\partial t}\right]\right)
$$

$$
\therefore f=\int_{0}^{\infty}\left(\lim _{n \rightarrow \infty}\left\{\frac{\partial\left(\log \left(x_{i}\right)_{n}\right)}{\partial t}\right\} \Delta T_{n}\right)
$$

Thus, from the supported equation of 5. The functional distribution of feature via each set of coordination is independent mapped and relevant features are extracted. This extraction is pressed to generate a data map (D) as shown in Eq. 6

$$
D=\Delta T\left\{\sum_{i=0}^{n}\left[\sum_{j=i+1}^{\infty} \frac{\partial(f)_{j}}{\partial t} \Delta T_{i}\right]\right\}
$$


The generated data maps $(D)$, testify the operation of extracting and co-laterally mapping the attributes with respect to feature set $(f)$ and parameter trained functions values $(T)$. The operation of Eq. 6 assures the coordination of collaterally distant variables. These processes help in generating an interdependent variable of threshold. These threshold values are represented by $(\Delta T)$ in Eq. 6 with a regionally evaluated matrix function.

\section{- Extraction and Prediction Model of DR}

The threshold values and DR based extracted values of feature set is collaboratively evaluated to form a regional extraction matrix of diabetic retinopathy. These values are bounded with Eq. 6 and Eq. 7 respectively in generating a prediction model $(P)$.

$$
P=\left[\frac{P(T)-P(F)}{P(T F)} * \frac{P(T)+P(F)}{P(F)}\right]
$$

$$
\therefore P=\int_{0}^{\infty} \log _{2} x_{i} \oplus \Delta T_{i} / \forall i \in\left(\frac{\partial D}{\partial t}\right)
$$

The Eq. 8 pre-dominantly expands the values set of evaluation and decision making towards ordering extraction and prediction of values towards a stream of decision making in categorizing the diabetic retinopathy $(D)$.

\section{DISCUSSION AND RESULTS}

The proposed article is developed and researched to extract the DR ratio of prediction and decision making. The results of $98.7 \%$ is based on extraction and validated in Table 1 with reference to feature set extraction and prediction. The inter-collateral values of such attributes are streamlined and processed with parametric features set of prediction $(P)$ as shown in Eq. 8

Table 1: Evaluation and Prediction of CAE Technique

\begin{tabular}{|l|c|c|c|c|c|c|}
\hline \multicolumn{2}{|c|}{ Feature set } & \multicolumn{2}{c|}{ Performance Estimation } & \multicolumn{2}{c|}{ Prediction Model (P) } \\
\hline $\begin{array}{c}\text { Dimension } \\
\text { Type }\end{array}$ & $\begin{array}{c}\text { Data } \\
\text { Type }\end{array}$ & $\begin{array}{c}\text { Streaming } \\
\text { values }\end{array}$ & $\begin{array}{c}\text { Pattern } \\
\text { size (MB) }\end{array}$ & $\begin{array}{c}\text { Features } \\
\text { extracted }\end{array}$ & $\begin{array}{c}\text { Typical } \\
\text { efficiency }\end{array}$ & $\begin{array}{c}\text { Proposed } \\
\text { CAE } \\
\text { efficiency }\end{array}$ \\
\hline $\begin{array}{l}\text { Image } \\
\text { (X-Ray) }\end{array}$ & $\begin{array}{l}\text { *.JPEG / } \\
\text { *.PNG / } \\
\text { *.BIN }\end{array}$ & 89.23 & 8972 & 73 & 91.3 & 92.98 \\
\hline $\begin{array}{l}\text { Image } \\
\text { (CT) }\end{array}$ & $\begin{array}{l}\text { *.JPEG / } \\
\text { *.PNG / } \\
\text { *.BIN }\end{array}$ & 90.23 & 90213 & 212 & 94.2 & 93.7 \\
\hline $\begin{array}{l}\text { Image } \\
\text { (MRI) }\end{array}$ & $\begin{array}{l}\text { *.JPEG / } \\
\text { *.PNG / }\end{array}$ & 89.87 & 67221 & 397 & 87.26 & 89.17 \\
\hline
\end{tabular}




\begin{tabular}{|c|c|c|c|c|c|c|}
\hline & $* . \mathrm{BIN}$ & & & & & \\
\hline $\begin{array}{l}\text { Image } \\
\text { (Thermal) }\end{array}$ & $\begin{array}{l}\text { *.JPEG } / \\
\text { *.PNG / } \\
\text { *.BIN }\end{array}$ & 92.10 & 32112 & 189 & 97.37 & 98.34 \\
\hline $\begin{array}{l}\text { Image } \\
\text { (other) }\end{array}$ & $\begin{array}{l}* . J P E G \quad / \\
* . P N G \quad / \\
* . B I N\end{array}$ & 82.11 & 18365 & 89 & 82.11 & 82.32 \\
\hline
\end{tabular}

\section{CONCLUSION}

The proposed system has successfully discussed on the functional attributes of classifying and categorizing the diabetic's retinopathy. The system is supported via a collateral attributes extraction and decision making. The technique has generated a schematic of data-maps (D) such that, each element of data maps are inter-linked to the attributes set (S) of dependency matrix. The process of evaluation is further discussed and featured in generating a feature set and thus expanding the attribute set. The proposed technique has successfully classified and predicted the occurrence of diabetic retinopathy with reference to open database of validation. The proposed technique has gained a performance efficiency of $98.7 \%$ with reference to datasets and validation through data maps. In near future, the process can be integrated with dynamic scheduling of information and processing unit via IoT enabled ecosystem for predictive framework modeling and evaluation.

\section{References}

[1]. Website: https://www.livemint.com/science/health/government-survey-found-11-8-prevalenceof-diabetes-in-india-11570702665713.html accessed on 12th March 2021 at 2:83 IST

[2]. Wu, L., Fernandez-Loaiza, P., Sauma, J., Hernandez-Bogantes, E., \& Masis, M. (2013). Classification of diabetic retinopathy and diabetic macular edema. World journal of diabetes, 4(6), 290.

[3]. Moss, S. E., Meuer, S. M., Klein, R., Hubbard, L. D., Brothers, R. J., \& Klein, B. E. (1989). Are seven standard photographic fields necessary for classification of diabetic retinopathy?. Investigative ophthalmology \& visual science, 30(5), 823-828.

[4]. Ege, B. M., Hejlesen, O. K., Larsen, O. V., Møller, K., Jennings, B., Kerr, D., \& Cavan, D. A. (2000). Screening for diabetic retinopathy using computer based image analysis and statistical classification. Computer methods and programs in biomedicine, 62(3), 165-175.

[5]. Akram, M. U., Khalid, S., \& Khan, S. A. (2013). Identification and classification of microaneurysms for early detection of diabetic retinopathy. Pattern Recognition, 46(1), 107116.

[6]. Ahmed, S. T., \& Sankar, S. (2020). Investigative Protocol Design of Layer Optimized Image Compression in Telemedicine Environment. Procedia Computer Science, 167, 2617-2622.

[7]. Yip, M. Y., Lim, G., Lim, Z. W., Nguyen, Q. D., Chong, C. C., Yu, M., ... \& Ting, D. S. (2020). Technical and imaging factors influencing performance of deep learning systems for diabetic retinopathy. NPJ digital medicine, 3(1), 1-12.

[8]. Ahmed, S. T., Sandhya, M., \& Sankar, S. (2019). A dynamic MooM dataset processing under TelMED protocol design for QoS improvisation of telemedicine environment. Journal of medical systems, 43(8), 1-12. 